\title{
Creation of early maturing productive forms of cereals using the cell biotechnology and physiologically active compounds
}

\author{
Bishimbayeva N.K. ${ }^{1,2 *}$, Baymagambetova K. ${ }^{3}$, Chudinov V.A. ${ }^{4}$, Sereda G.A. ${ }^{3}$, \\ Gass O.S. ${ }^{5}$, Bekenova L.V. ${ }^{6}$, Begzat A.N. ${ }^{1}$, Ertayeva B.Y. ${ }^{1,8}$, Karabayev M.K. ${ }^{8}$, \\ Urozaliyev R.A. ${ }^{3}$ \\ ${ }^{1}$ Al-Farabi Kazakh National University, Almaty, Kazakhstan \\ ${ }^{2}$ Kazakh National Agrarian University, Almaty, Kazakhstan \\ ${ }^{3}$ Kazakh Research Institute of Agriculture and Plant Growing, Almalybak, Kazakhstan \\ ${ }^{4}$ Karabalyk Agricultural Experimental Station, Kostanai region, Kazakhstan \\ ${ }^{5}$ Pavlodar Research Institute of Agriculture, Kazakhstan \\ ${ }^{6}$ Karaganda Research Institute of Breeding and Crop Production, Kazakhstan \\ ${ }^{7}$ North-Kazakhstan experimental station, North Kazakhstan region, Kazakhstan \\ ${ }^{8}$ Institute of Plant Biology and Biotechnology, Almaty, Kazakhstan \\ *e-mail: gen_jan@mail.ru
}

Creation of early maturing genotypes of main cereal crops - spring wheat and barley, is an actual task for northern regions of Kazakhstan. We have distinguished the increasing of phenotypic variability by two approaches: use of long-term plant regeneration cell technology (wheat) and using physiologically active compounds (wheat, barley), both elaborated in our laboratory of cell biology (Institute of Plant Biology and Biotechnology). Then, we have selected during several years forms with inherited signs of precocity and high productivity. Both approaches are genotype independent and can be used for any commercially important variety or line. It's noteworthy, that these approaches allowed to obtain principally new forms, incorporating traits (early maturity, high productivity, drought resistance, grain quality, etc.), simultaneous combination of which is difficult or impossible to achieve using the methods of traditional classical breeding or genetic transformation. Most of these signs are under polygenic control. Obtained data are discussed from the point of possible epigenetic mechanisms underlying the arising of new forms. Intense breeding has eroded genetic diversity, and epigenetic diversity now emerge as a new source of phenotypic variations to improve adaptation to changing environments and ensure the yield and quality of crops. Approaches we propose for enhancing phenotypic diversity can be well used in breeding programs. Obtained forms are the good models for transcriptome search of new genes responsible for phenotype reprogramming.

Acknowledgements: This work performed under the applied project GF1 No. 1911 (20122014), innovation grant NATD MIT RK (2011-2014), Target Programm of fundamental research No. 0149 (2015-2017), MES RK. 